\title{
Validation of multiple sclerosis diagnoses in the Swedish National Patient Register
}

\author{
Chantelle Murley $^{1}{ }^{[} \cdot$ Emilie Friberg $^{1} \cdot$ Jan Hillert $^{2} \cdot$ Kristina Alexanderson $^{1} \cdot$ Fei Yang $^{1}$
}

Received: 23 April 2019 / Accepted: 29 August 2019 / Published online: 6 September 2019

(c) The Author(s) 2019

\begin{abstract}
Population-based registers are widely used in epidemiological studies. We aimed to estimate the validity of multiple sclerosis (MS) diagnoses registered in the Swedish National Patient Register (NPR) by two sequential register-based case-definition algorithms. Prevalent MS patients aged 16-64 years were identified from the in- and specialised out-patient NPR in 20012013, using International Classification of Diseases code G35. These identified MS diagnoses were validated through two sequential register-based case-definition algorithms, as the 'gold-standard' reference, by linking individual-level data longitudinally to other nationwide registers. The primary algorithm first sought to corroborate the MS diagnoses with MS-specific information in other nationwide registers. The exploratory secondary algorithm identified individuals with MS-related information in other registers and those who were unable to be followed sufficiently. Through multi-register linkage, we estimated the number of confirmed and uncertain individuals with an MS diagnosis recorded in the NPR. A total of 19,781 individuals (mean age at first visit 45.2 years; $69.5 \%$ women) had at least one MS diagnosis recorded in the NPR during 2001-2013. Using the two case-definition algorithms, $92.5 \%(n=18,291)$ of the MS diagnoses recorded in the NPR were confirmed, while $7.5 \%(n=1490)$ remained uncertain. Our findings indicate that a very high percentage of patients coded with an MS diagnosis in the Swedish NPR actually have MS, and supports the use of the NPR as a viable source to identify individuals with an MS diagnosis for population-based research. This exploratory methods paper suggests an alternative novel method to verify individuals' diagnoses in register-based settings.
\end{abstract}

Keywords Validation studies $\cdot$ Electronic health records $\cdot$ Multiple sclerosis $\cdot$ Sick leave $\cdot$ Administrative data $\cdot$ Swedish National Patient Register

\section{Background}

The Swedish nationwide population-based registers are widely used in epidemiological studies, providing a less resource demanding data source than patient records to establish representative scientific results [1-4]. Nonetheless, administrative registers may be subject to diagnostic

Electronic supplementary material The online version of this article (https://doi.org/10.1007/s10654-019-00558-7) contains supplementary material, which is available to authorized users.

Chantelle Murley

chantelle.murley@ki.se

1 Division of Insurance Medicine, Department of Clinical Neuroscience, Karolinska Institutet, 17177 Stockholm, Sweden

2 Division of Neurology, Department of Clinical Neuroscience, Karolinska Institutet, 17177 Stockholm, Sweden and coding errors [5]. Individual-level linkage across the different registers can be used to corroborate the information contained within a single register. The National Patient Register (NPR), a principal data source for research [4, 5], contains records of healthcare visits in Sweden with diagnoses registered by the International Classification of Diseases (ICD) codes. The validity of the complete NPR is high [5], but knowledge of data quality is lacking for some specific diseases, such as multiple sclerosis (MS).

MS is a neurodegenerative disease that typically affects younger adults, causing increasing levels of cognitive and physical impairment as the disease progresses [6-8]. Sweden has an especially high prevalence [7]; with an estimated all-age nationwide MS prevalence of 189 per 100,000 [9]. Despite that MS diagnoses are now being set earlier and more accurately, no single clinical feature or diagnostic test can positively diagnose MS [10-12]. This clinical context may reduce our certainty of the recorded MS diagnoses due 
to the risk of misdiagnosis between MS and other degenerative neurological diseases, a risk which has been suggested to still exist with the 2017 update of the McDonald criteria $[10,13]$. Therefore, it has been questioned to what extent an MS diagnosis is incorrectly assigned and recorded in administrative records during the period investigating whether a patient has MS [10, 13-18].

Besides the NPR, several other Swedish nationwide registers contain MS-specific information (i.e., MS ICD codes or medications), which could be used to identify MS cases. Accordingly, we aimed to assess the validity of MS diagnoses recorded in the NPR with a novel method using information linked from other nationwide registers in two sequential register-based case-definition algorithms.

\section{Methods}

This validation study evaluates the NPR as a relevant source for MS studies by comparing the records of all individuals aged 16-64 with an MS code during 1 January 2001-31 December 2013 in the NPR against other register-based MS records, through two sequential register-based case-definition algorithms as the 'gold-standard' reference.

\section{Data sources}

The unique personal identity number assigned to all residents in Sweden enabled individual-level linkages across six nationwide registers [19], administered by the following four authorities:

- National Board of Health and Welfare: The National Patient Register (NPR) includes information about healthcare visits, including main and all side diagnoses coded according to the Swedish version of the ICD at time of visit [20]. The NPR has had nationwide coverage since 1987 of in-patient admissions and of out-patient specialist visits since 2001 [5]. The Swedish Prescribed Drug Register (SPDR) contains nationwide records of dispensed medication prescriptions with Anatomical Therapeutic Classification (ATC) codes since 1 July 2005 [21]. The Cause of Death Register (CDR) contains nationwide information on the date, underlying and contributory causes of death recorded by ICD codes since 1961 [22].

- Karolinska University Hospital: The Swedish MS Register (SMSReg) is a nationwide but voluntary MSspecific clinical register that is used for nationwide pharmacological surveillance and for enhancing quality of care [23, 24]. SMSReg contains comprehensive clinical information [25] for included patients diagnosed with MS to support clinical decision-making, including retro- spective information predating the SMSReg's establishment in 2001 [26]. The accuracy and completeness of the clinical data has been recently estimated to be of value for future studies [25].

- Social Insurance Agency: The Micro-Data for Analysis of the Social Insurance System (MiDAS) contains information on sickness absence (since 2005) and disability pension benefits (since 1994) (dates and diagnoses (by ICD codes)) [27].

- Statistics Sweden: The Longitudinal Integration Database for Health Insurance and Labour Market Studies (LISA), comprises annual socio-demographic information on all people registered as living in Sweden [28, 29].

\section{Study population}

All individuals with a main or side diagnosis of MS (ICD-10: G35) recorded when having a healthcare visit in 2001-2013, aged 16-64 years, were identified in the NPR $(n=19,781)$. From the date of first MS diagnosis in 2001-2013 in the NPR, both retrospective and prospective information was obtained from the above mentioned six registers, in order to confirm the MS diagnosis. If it was not possible to confirm the MS diagnosis, the individual was followed until emigration, death, or end of data extraction of each register (varies between 2013 and 2016), whichever came first (Fig. 1).

\section{Statistical analyses}

We first described the MS patients in terms of frequencies and percentages by: sex (women/men); healthcare setting (in-/out-patient); whether MS was the main diagnosis for the first record with an MS diagnosis during 2001-2013 (yes/ no); number of visits with an MS diagnosis recorded in the NPR ever $(<3 / \geq 3)$; whether in the NPR prior to 2001 with an MS diagnosis (yes/no); whether died before 31 December 2013 (yes/no); and age at first record with MS in the NPR during 2001-2013.

The annual MS prevalence (total and sex-specific) were estimated from 2001 to 2013 of all individuals aged 16-64 at the time of MS diagnosis according to the NPR. The prevalence estimates were expressed per 100,000 of the total population in Sweden aged 16-64, identified in LISA for the respective year.

\section{Validation}

Two sets (primary and secondary) of register-based casedefinition algorithms were constructed by the multidisciplinary research group for this study to validate MS diagnoses recorded in the NPR with the extensive register data available. The algorithms cross-checked the MS diagnoses at an individual-level with specific information on MS from all 


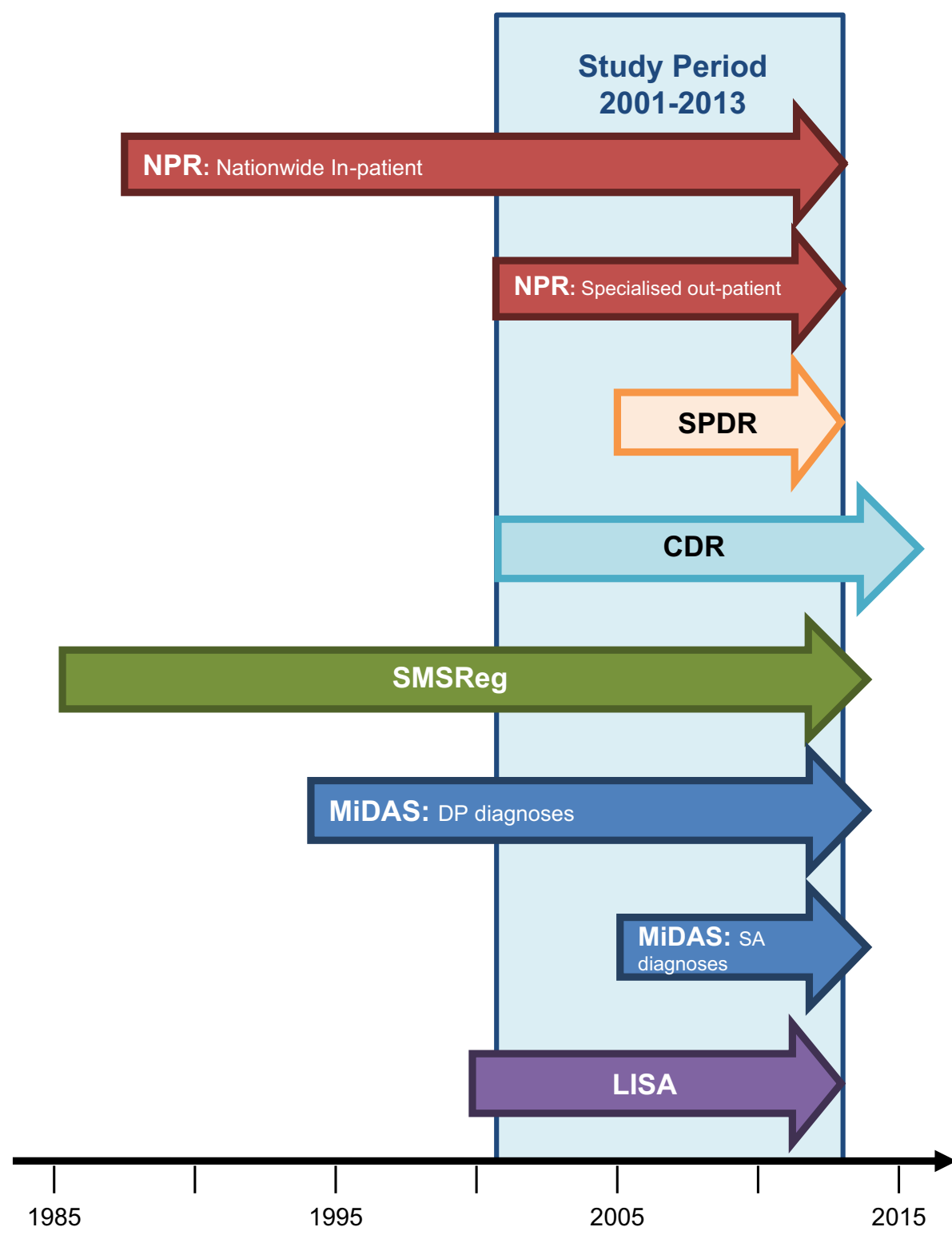

Fig. 1 Data coverage and availability from the nationwide register sources in relation to the study period. Notes The study period refers to dates to identify MS cases from the Swedish National Patient Register (NPR) among people aged 16-64 at the time of the visit. Six nationwide registers containing individual-level data were used. The NPR (red arrows) contains information healthcare visits according to ICD codes, but with different coverage dates with regards to the healthcare setting. MS healthcare visits in both the in-patient (nationwide since 1987) and specialised out-patient (included 2001) healthcare settings until 31 December 2013 were included. The Swedish Prescribed Drug Register (SPDR) (orange arrow) contains information of the nationwide records of dispensed medication prescriptions with Anatomical Therapeutic Classification (ATC) codes since 1 July 2005 and were available until 31 December 2013. The Cause of Death Register (CDR) (aqua arrow) contains nationwide information since 1961 on the date and underlying cause of death, including contributory causes, according to ICD codes. ICD codes were not available after 2016. The nationwide voluntary clinical quality register, Swedish MS Register (SMSReg) (green arrow), was established in
2001 and contains comprehensive clinical information of MS-related care for the included patients, including retrospective information predating the SMSReg's creation from selected neurology clinics and was available until September 2014. Micro-Data for Analysis of the Social Insurance System (MiDAS) (blue arrows) has coverage of diagnoses for disability pension (DP) benefits from 1994 and diagnoses for sickness absence (SA) benefits from 2005, and was available until 2014. Longitudinal Integration Database for Health Insurance and Labour Market Studies (LISA) (purple arrow) contains annual individual-level information about the socio-demographics of the total population registered as resident in Sweden, as of 31 December, and was used for the years 2000-2013. ATC Anatomical Therapeutic Classification, $C D R$ Cause of Death Register, $D P$ disability pension, ICD International Classification of Diseases, LISA Longitudinal Integration Database for Health Insurance and Labour Market Studies, MiDAS Micro-Data for Analysis of the Social Insurance System, $M S$ multiple sclerosis, NPR National Patient Register, SA sickness absence, SPDR Swedish Prescribed Drug Register, SMSReg Swedish MS Register 
available nationwide register sources in Sweden, including prior to and after 2001-2013 (Fig. 1). These algorithms, applied in a sequential manner, were thought to be equivalent to a diagnostic test to confirm the registered diagnoses [30]. Accordingly, the MS diagnoses recorded in the NPR were treated as 'provisional' MS until validated as "confirmed' MS as per the algorithms.

Figure 2 displays the primary and secondary sets of case-definition algorithms, including the order of the steps identifying confirmed MS and the 'gold-standard' data source used [1]. The primary algorithm identified MS-specific codes (ICD-10 G35, ICD-9 340, or specific ATC codes for MS medications) in other registers in six steps. In the first step of this algorithm, individuals with clinical MS information, entered by their treating neurologist, in the SMSReg were identified. The secondary casedefinition was then applied to the remaining provisional MS diagnoses after completing the primary algorithm. This exploratory algorithm matched to other MS-related information (MS-like symptom ICD codes), and sought to identify individuals who were unable to be sufficiently followed in the data to confirm the diagnosis as 'plausible'
MS. The individuals who remained at the end of the last step in each set of case-definition algorithms were classified as 'uncertain' MS (i.e., patients who received at least one MS diagnosis recorded in the NPR, but without other register-based information corroborating the diagnosis). The confirmed MS are those identified according to both case-definition algorithms, i.e., had other register-based MS information supporting their MS diagnosis in the NPR. We then plotted the proportions of confirmed, plausible, and uncertain MS by the year of first MS record in the NPR.

Finally, in order to estimate the influence of the uncertain MS cases in the NPR if included in study populations, we profiled the descriptive characteristics of those remaining as uncertain MS after each set of algorithms and those confirmed MS (including plausible MS diagnoses). Similarity of the characteristics of uncertain MS after the secondary algorithm and those of confirmed MS were tested using Chi square tests. The mean number of years between the first and second MS record in the NPR with $95 \%$ confidence intervals (CI) were calculated. All analyses were conducted in SAS v.9.4.

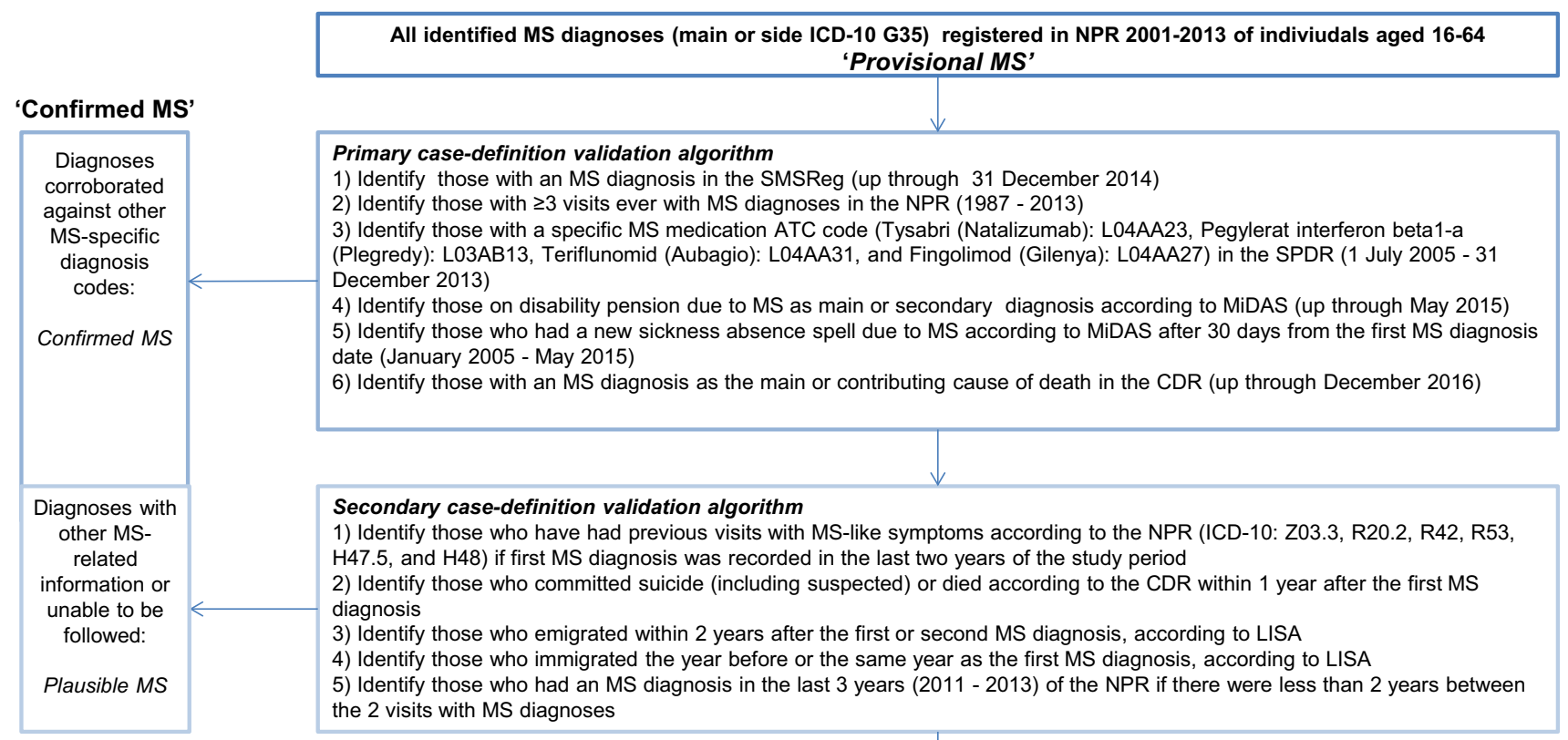

'Uncertain MS'

Cases unable to be corroborated by the algorithms

Fig. 2 Primary and secondary case-definition algorithms which together form the 'gold standard' to identify the confirmed MS diagnoses registered in the Swedish National Patient Register (NPR) by validating against individual-level information from other register data sources. Notes Given that patients with clinically isolated syndrome (CIS) should be kept as uncertain MS, and may be treated with a subset of DMTs, the following DMTs and ATC codes were not included in the algorithm: Interferon beta 1-a: L03AB07, Interferon beta 1-b: L03AB08, and Glatiramercetat: L03AX13. ATC Anatomical Therapeutic Classification, CDR Cause of Death Register, $C I S$ clinically isolated syndrome, $D M T$ disease modifying therapies, $I C D$ International Classification of Disease, LISA Longitudinal Integration Database for Health Insurance and Labour Market Studies, MiDAS Micro-Data for Analysis of the Social Insurance System, $M S$ multiple sclerosis, NPR National Patient Register, SPDR Swedish Prescribed Drug Register, SMSReg Swedish MS Register 


\section{Compliance with ethical standards}

This project was approved by the Regional Ethical Review Board of Stockholm in accordance with the Declaration of Helsinki.

\section{Results}

Overall, 19,781 individuals (69.5\% women) with MS diagnoses recorded in the NPR when aged 16-64 were identified in 2001-2013. In all, $81.7 \%(n=16,158)$ were first identified from specialised out-patient visits and $89.7 \%(n=17,738)$ had MS as the main diagnosis. They together had 308,761 visits with MS as a listed diagnosis during 2001-2013.

The nationwide prevalence of MS according to the NPR in the population aged 16-64 years steadily increased over the study period (Fig. 3). In 2013, the estimated prevalence was 258.3 (women 369.1; men 150.8) per 100,000. Women had a higher prevalence than men throughout the study.

Of the identified individuals, $92.5 \%(n=18,291)$ had their diagnosis confirmed by the validation algorithms (Table 1). The primary algorithm confirmed the majority of diagnoses $(17,922 ; 90.6 \%)$, with fewer corroborated by the secondary algorithm $(369 ; 1.9 \%)$. We classified $1490(7.5 \%)$ individuals as uncertain MS after cross-checking both casedefinition algorithms. After the first two steps of the primary algorithm, MS diagnosis in the SMSReg and $\geq 3$ visits due to MS recorded in the NPR, $89.4 \%(n=17,692)$ of the provisional MS diagnoses were confirmed.

The profiles of the confirmed and uncertain MS are presented in Table 2. The mean number of years between the first and second visit (2001-2013) in the NPR for the uncertain MS were 0.85 (95\% CI 0.70-1.00) after the primary case-definition algorithm and 1.01 (95\% CI 0.78-1.24) after the secondary case-definition algorithm. Of the individuals with confirmed MS $(n=18,291), 16,527$ (90.4\%) had MS as the main diagnosis and $14,921(81.6 \%)$ first presented to out-patient healthcare. Of the uncertain MS, most of them had only one visit due to an MS diagnosis recorded in the NPR $(78.5 \%, \mathrm{n}=1169)$ (not presented in the table).

A peak in first time NPR-registered MS diagnoses was observed when out-patient healthcare was included in 2001 (Online Resource 1).

\section{Discussion}

In this study we identified all individuals aged 16-64 with MS as a main or side diagnosis in the Swedish NPR during 2001-2013. We compared these provisional MS diagnoses against individual-level MS-specific information in other nationwide registers. With this novel method, we confirmed that $92.5 \%$ of the NPR-registered MS diagnoses

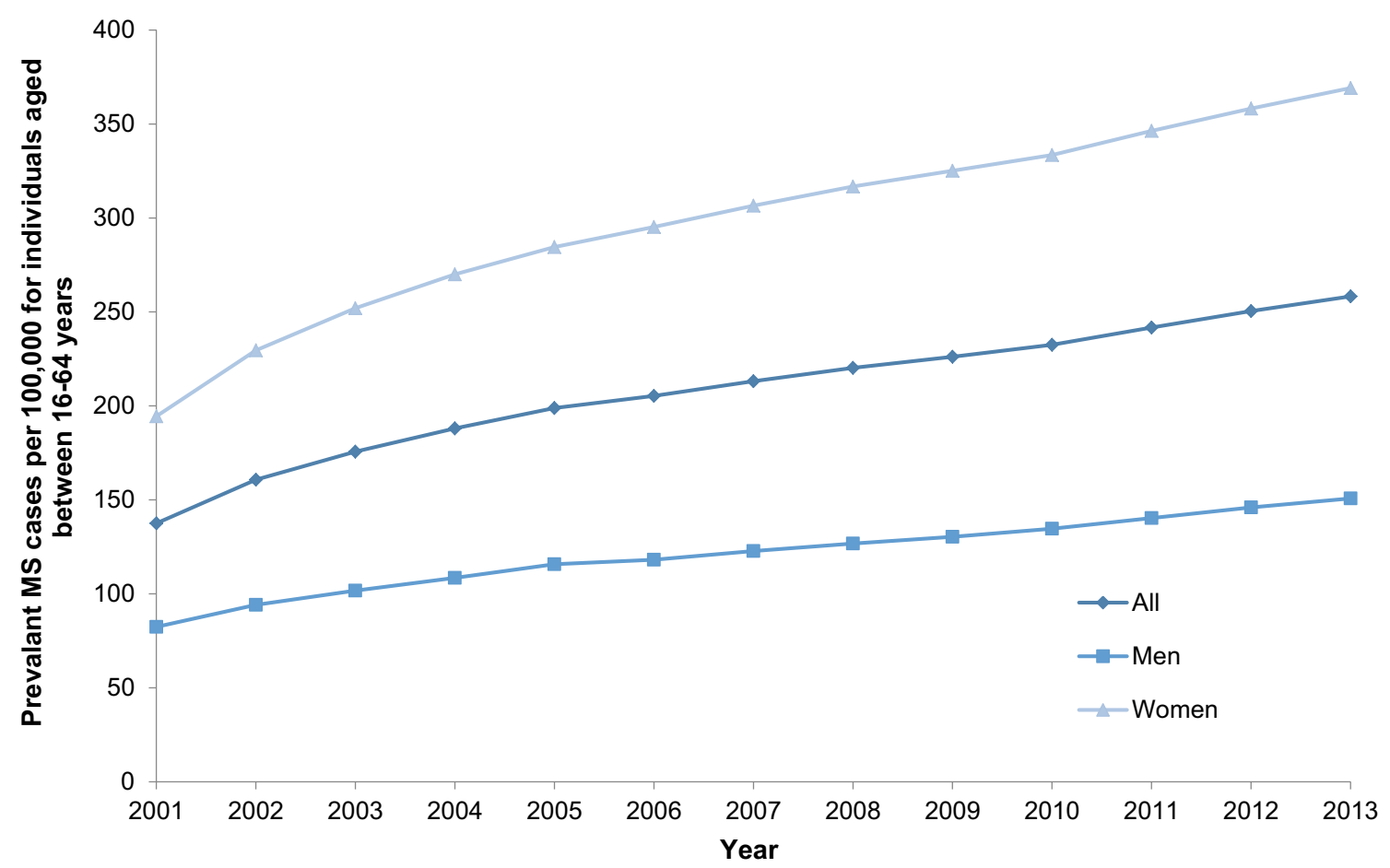

Fig. 3 Prevalence of MS per 100,000 individuals aged 16-64 in 2001-2013, identified in the Swedish National Patient Register. MS multiple sclerosis 
Table 1 Steps in the process to validate the MS diagnoses recorded in the Swedish National Patient Register $($ NPR $)(2001-2013)(n=19,781)^{\text {a }}$ by two sequential register-based case-definition algorithms

$\begin{array}{ll}\mathrm{N} \text { identified } & \mathrm{N} \text { remaining (i.e., } \\ \text { (i.e., confirmed } & \text { uncertain } \mathrm{MS} \text { ) } \\ \mathrm{MS}) & \end{array}$

Primary case-definition algorithm

(1) Identify those with an MS diagnosis in the SMSReg (up through 31 December 2014)

$\begin{array}{rl}14,033 & 5748 \\ 3659 & 2089 \\ 6 & 2083\end{array}$

(3) Identify those with a specific MS medication ATC code (Tysabri (Natalizumab): L04AA23, Pegylerat interferon beta1-a (Plegredy): L03AB13, Teriflunomid (Aubagio): L04AA31, and Fingolimod (Gilenya): L04AA27) in the SPDR (1 July 2005-31 December 2013)

(4) Identify those on disability pension due to MS as main or secondary diagnosis according to MiDAS (up through May 2015)

(5) Identify those who had a new sickness absence spell due to MS according to MiDAS after 30 days from the first MS diagnosis date (January 2005-May 2015)

(6) Identify those with an MS diagnosis as the main or contributing cause of death in the CDR (up through December 2016)

Subtotals from the primary case-definition algorithm

Secondary case-definition algorithm

(1) Identify those who have had previous visits with MS-like symptoms according to the NPR (ICD-10: Z03.3, R20.2, R42, R53, H47.5, and H48) if the first MS diagnosis was recorded in the last 2 years of the study period ${ }^{\mathrm{b}}$

(2) Identify those who committed suicide (including suspected) or died according to the CDR within 1 year after the first MS diagnosis

(3) Identify those who emigrated within 2 years after the first or second MS diagnosis, according to LISA

(4) Identify those who immigrated the year before or the same year as the first MS diagnosis, according to LISA

(5) Identify those who had an MS diagnosis in the last 3 years (2011-2013) of the NPR if there were less than 2 years between the 2 visits with MS diagnoses

\section{Subtotals from the secondary case-definition algorithm}

Total

$\begin{array}{ll}158 & 1925 \\ 51 & 1874 \\ 15 & 1859\end{array}$

1859

ATC Anatomical Therapeutic Classification, CDR Cause of Death Register, ICD International Classification of Diseases, LISA Longitudinal Integration Database for Health Insurance and Labour Market Studies, MiDAS Micro-Data for Analysis of the Social Insurance System Register, MS multiple sclerosis, NPR National Patient Register, SPDR Swedish Prescribed Drug Register, SMSReg Swedish MS Register

${ }^{a}$ All individuals with a record of an MS diagnosis registered during 1 January 2001 and 31 December 2013 as per ICD-10 (G35) in the Swedish National Patient Register and aged 16-64 at first diagnosis in the study period

${ }^{\mathrm{b}}$ Z03.3: Observation for suspected nervous system disorder, R20.2: Paraesthesia of skin, R42: Dizziness and giddiness, R53: Malaise and fatigue, H47.5: Disorders of other visual pathways, and H48: Disorders of optic nerve and visual pathways in diseases classified elsewhere

could be validated after the two sequential register-based case-definition algorithms. Our findings support the NPR as a valuable source to identify MS cases in epidemiological studies.

The majority of provisional and confirmed MS diagnoses first presented in out-patient settings, with MS as the main diagnosis. A slightly higher percentage of the uncertain MS diagnoses were a side diagnosis. The spike in the number of registered MS diagnoses in 2001 corresponds with the inclusion of the specialised out-patient visits, and tapers off by 2004 to reasonably consistent annual numbers of first time identified diagnoses for the remaining study years. This trend suggests caution is required when identifying newly diagnosed MS cases from the NPR prior to 2004, i.e., the first years of including out-patient visits in the NPR.
The first two steps of the primary case-definition algorithm (MS diagnosis in the SMSReg and $\geq 3$ visits with MS ever in the NPR) confirmed the majority of provisional MS diagnoses. The exploratory secondary case-definition algorithm identified a small number of plausible MS diagnoses, predominantly in the later years, with insufficient follow-up time to reasonably identify MS information in the registers. The individuals with uncertain MS after both case-definition algorithms could plausibly represent patients who were initially suspected of having MS, but the diagnosis was never confirmed, or simply due to administrative error. In total, $78.5 \%$ of the uncertain MS only had one visit ever due to MS.

A previous cross-referencing of the SMSReg to NPR by the National Board of Health and Welfare found that 
Table 2 Characteristics of individuals identified during 1 January 2001-31 December 2013 with an MS diagnosis registered in the Swedish National Patient Register (NPR) ${ }^{\mathrm{a}}$ when aged 16-64, according to the register-based case-definition algorithms

\begin{tabular}{|c|c|c|c|c|c|c|c|c|c|}
\hline & \multirow{2}{*}{\multicolumn{2}{|c|}{$\begin{array}{l}\text { Provisional MS } \\
\text { diagnoses in the } \\
\text { NPR } \\
\mathrm{n}=19,781\end{array}$}} & \multirow{2}{*}{\multicolumn{2}{|c|}{$\begin{array}{l}\text { Uncertain MS } \\
\text { as per Primary } \\
\text { case-definition } \\
\text { algorithm } \\
\mathrm{n}=1859^{\mathrm{b}}\end{array}$}} & \multirow{2}{*}{\multicolumn{2}{|c|}{$\begin{array}{l}\text { Uncertain MS as } \\
\text { per Secondary } \\
\text { case-definition } \\
\text { algorithm } \\
\mathrm{n}=1490^{\mathrm{b}}\end{array}$}} & \multirow{2}{*}{\multicolumn{2}{|c|}{$\begin{array}{l}\text { Confirmed MS as } \\
\text { per both case-defi- } \\
\text { nition algorithms } \\
\\
\mathrm{n}=18,291\end{array}$}} & \multirow{3}{*}{$\begin{array}{l}\chi^{2} \text { test } \\
p \text { value }^{\mathrm{c}}\end{array}$} \\
\hline & & & & & & & & & \\
\hline & $\mathrm{n}$ & $\%$ & $\mathrm{n}$ & $\%$ & $\mathrm{n}$ & $\%$ & $\mathrm{n}$ & $\%$ & \\
\hline \multicolumn{9}{|l|}{ Sex } & $<0.001$ \\
\hline Women & 13,743 & 69.5 & 1224 & 65.8 & 970 & 65.1 & 12,773 & 69.8 & \\
\hline Men & 6038 & 30.5 & 635 & 34.2 & 520 & 34.9 & 5518 & 30.2 & \\
\hline \multicolumn{7}{|l|}{$\begin{array}{l}\text { Healthcare setting of first recorded visit in the } \\
\text { NPR with MS }\end{array}$} & & & 0.166 \\
\hline In-patient Register & 3623 & 18.3 & 322 & 17.3 & 253 & 17.0 & 3370 & 18.4 & \\
\hline Out-patient Register & 16,158 & 81.7 & 1537 & 82.7 & 1237 & 83.0 & 14,921 & 81.6 & \\
\hline Main diagnosis as MS for the first recorded visit & 17,738 & 89.7 & 1520 & 81.8 & 1211 & 81.3 & 16,527 & 90.4 & $<0.001$ \\
\hline \multicolumn{9}{|l|}{ Number of visits in the NPR with MS ever ${ }^{\mathrm{d}}$} & $<0.001$ \\
\hline$<3$ & 2834 & 14.3 & 1859 & 100.0 & 1490 & 100.0 & 1344 & 7.3 & \\
\hline$\geq 3$ & 16,947 & 85.7 & $\mathrm{c}$ & $\mathrm{c}$ & $\mathrm{c}$ & $\mathrm{c}$ & 16,947 & 92.7 & \\
\hline In the NPR prior to 1 January 2001 with MS & 4399 & 22.2 & 29 & 1.6 & 28 & 1.9 & 4371 & 23.9 & $<0.001$ \\
\hline Died (all-cause) during 2001-2013 & 1806 & 9.1 & 96 & 5.2 & 65 & 4.4 & 1741 & 9.5 & $<0.001$ \\
\hline \multicolumn{10}{|c|}{ Age at first record with MS in the NPR during 2001-2013 } \\
\hline $16-20$ & 508 & 2.6 & 42 & 2.26 & 31 & 2.08 & 477 & 2.61 & \\
\hline $21-25$ & 1072 & 5.4 & 91 & 4.90 & 73 & 4.90 & 999 & 5.46 & \\
\hline $26-30$ & 1813 & 9.2 & 168 & 9.04 & 127 & 8.52 & 1686 & 9.22 & \\
\hline $31-35$ & 2138 & 10.8 & 202 & 10.87 & 155 & 10.40 & 1983 & 10.84 & \\
\hline $36-40$ & 2397 & 12.1 & 201 & 10.81 & 174 & 11.68 & 2223 & 12.15 & \\
\hline $41-45$ & 2686 & 13.6 & 219 & 11.78 & 173 & 11.61 & 2513 & 13.74 & \\
\hline $46-50$ & 2621 & 13.3 & 234 & 12.59 & 181 & 12.15 & 2440 & 13.34 & \\
\hline $51-55$ & 2731 & 13.8 & 243 & 13.07 & 201 & 13.49 & 2530 & 13.83 & \\
\hline $56-60$ & 2451 & 12.4 & 273 & 14.69 & 224 & 15.03 & 2227 & 12.18 & \\
\hline $61-64$ & 1364 & 6.9 & 186 & 10.01 & 151 & 10.13 & 1213 & 6.63 & \\
\hline
\end{tabular}

ICD International Classification of Diseases, $M S$ multiple sclerosis, NPR National Patient Register

${ }^{a}$ Identified by ICD-10 code G35 registered as the main or side diagnosis for in-patient and specialised out-patient care in the NPR. Inclusion criteria: All individuals with a record of a prevalent MS diagnosis registered in the Swedish NPR and aged 16-64 years at the time of the first code appearing during the study period 1 January 2001 and 31 December 2013, n = 19,781

${ }^{b} \mathrm{n}=369$ individuals were plausible MS as per the second case-definition algorithm and thus many individuals in the primary and secondary algorithm groups overlap

${ }^{\mathrm{c}}$ Significantly different proportions between the confirmed MS (both algorithms) and uncertain MS as per secondary algorithm, as tested by Chi square tests with alpha set at 0.05

${ }^{\mathrm{d}}$ Step 3 of the primary-case definition algorithm was to identify individuals with $>3$ visits ever recorded in the NPR with MS as confirmed MS

only $4 \%$ of MS entries in the NPR in 2008 were coded as 'possible MS' in the SMSReg [26], suggesting high validity of MS diagnoses recorded in the NPR [26]. Accordingly, when selecting a study population, the voluntary SMSReg may underestimate the MS population, especially for particular geographical regions [26], and we, based on this study, add that the mandated NPR may slightly overestimate the MS population $(<10 \%)$. Further studies are needed to elucidate occurrence of this.
Our prevalence estimates of MS for 16-64 year olds were higher than an all-age estimate of 189 per 100,000 at 31 December 2008 with MS identified in either the SMSReg or NPR [9]. The improved diagnostic criteria, better awareness, more efficacious disease modifying therapies (DMTs), and ultimately extended survival could be a potential explanation $[6,7]$. The high prevalence of MS among working-aged individuals and increasing treatment options necessitates representative real-world results [6], which the NPR enables [1,2]. 
Strengths of this study include the numerous nationwide registers with MS-specific information available to corroborate the MS diagnoses in the NPR. Usually, a 'goldstandard' of disease is derived from clinical data, albeit for a sample of the total population. Instead, we used a novel method of register-based algorithms linking individuallevel register data, including MS-specific clinical information from the SMSReg [25], to determine the validity of all identified MS cases in Sweden in 2001-2013. This study included years both before and after identification in the NPR, a design consistent with the chronic and progressive nature of MS [31] and treatment guidelines for annual healthcare visits for individuals with MS or clinically isolated syndrome (CIS) [32].

One limitation was that the case-definition algorithms were limited to the different coverage dates of the contributing registers. For example, the SPDR, which was available for July 2005-December 2013 and only covered dispensed prescribed drugs, therefore, excluding both indented drugs administrated at clinics and DMTs that became available after 2013. Further, both MS and CIS patients may be prescribed interferon beta therapies [32], therefore, these ATC codes could not be used to confirm MS diagnoses.

In conclusion, the certainty of MS diagnoses for patients aged 16-64 in the NPR is very high according to this validation using register-based algorithms. These findings strengthen the notion that the Swedish NPR is a valuable source for MS populations in nationwide epidemiological studies.

Acknowledgments Open access funding provided by Karolinska Institutet.

Author contributions All authors (CM, EF, JH, KA, and FY) participated in the design of the study. FY performed data management, and $\mathrm{CM}, \mathrm{FY}$, and EF were involved in the data analyses. All authors (CM, EF, JH, KA, and FY) contributed to the interpretation of results, and participated in the writing and reviewing of the drafts, including approval of the final version of the manuscript.

Funding This work was supported by a grant from the Swedish Research Council for Health, Working Life and Welfare (Dnr: 20071762) and by an unrestricted research grant from Biogen. For both, Kristina Alexanderson is the Principal Investigator of the grants. The design of the study, data collection, analyses, interpretations of data, and writing of manuscript were performed without involvement of the funding body.

Data/code explanation Not applicable. The data cannot be made publically available due to privacy regulations. The data used in this study is administered by the Division of Insurance Medicine, Karolinska Institutet. According to the General Data Protection Regulation, the Swedish law SFS 2018:218, the Swedish Data Protection Act, the Swedish Ethical Review Act, and the Public Access to Information and Secrecy Act, these types of sensitive data can only be made available, after legal review, for researchers who meet the criteria for access to this type of sensitive and confidential data. Readers may contact Professor Kristina Alexanderson (kristina.alexanderson@ki.se) regarding the data.

\section{Compliance with ethical standards}

Conflict of interest The authors declare the following interests based on ICMJE recommendations with respect to the research, authorship, and/or publication of this article: $\mathrm{CM}, \mathrm{EF}$, and FY received salaries partly funded by an unrestricted research grant from Biogen. KA has received unrestricted research grants from Biogen. $\mathrm{JH}$ has received honoraria for serving on advisory boards or steering committees for Biogen, Merck, Sanofi-Genzyme and Novartis and speaker's fees from Biogen, Novartis, Merck-Serono, Bayer-Schering, Teva and SanofiGenzyme. He has served as Principal Investigator for projects, or received unrestricted research support from, BiogenIdec, Merck-Serono, TEVA, Sanofi-Genzyme and Bayer-Schering and his research is also supported by the Swedish Research Council and the Swedish Brain Foundation.

Ethical approval Ethical approval for the project was granted by the Regional Ethical Review Board of Stockholm, Sweden (Dnr: 2007/762$31 ; 2009 / 23-32 ; 2009 / 1917-32 ; 2011 / 1710-32 ; 2012 / 863-32$; and 2014/236-32), in accordance with the Declaration of Helsinki.

Informed consent Due to the register-based nature of this project and given that all data were anonymised when delivered to us, we do not have information about the identity of those included in analyses. Accordingly, there was no informed consent from the patients involved, in line with the Ethical approval.

Open Access This article is distributed under the terms of the Creative Commons Attribution 4.0 International License (http://creativeco mmons.org/licenses/by/4.0/), which permits unrestricted use, distribution, and reproduction in any medium, provided you give appropriate credit to the original author(s) and the source, provide a link to the Creative Commons license, and indicate if changes were made.

\section{References}

1. Sorensen HT, Sabroe S, Olsen J. A framework for evaluation of secondary data sources for epidemiological research. Int J Epidemiol. 1996;25(2):435-42.

2. Olsen J. Register-based research: some methodological considerations. Scand J Public Health. 2011;39(3):225-9. https://doi. org/10.1177/1403494811402719.

3. Wennman-Larsen A, Nilsson MI, Saboonchi F, Olsson M, Alexanderson K, Fornander T, et al. Can breast cancer register data on recommended adjuvant treatment be used as a proxy for actually given treatment? Eur J Oncol Nurs. 2016;22:1-7. https://doi. org/10.1016/j.ejon.2016.02.010.

4. Ludvigsson JF, Almqvist C, Bonamy A-KE, Ljung R, Michaëlsson K, Neovius M, et al. Registers of the Swedish total population and their use in medical research. Eur J Epidemiol. 2016;31(2):125-36.

5. Ludvigsson JF, Andersson E, Ekbom A, Feychting M, Kim JL, Reuterwall C, et al. External review and validation of the Swedish national inpatient register. BMC Public Health. 2011;11:450. https ://doi.org/10.1186/1471-2458-11-450.

6. Giovannoni G, Butzkueven H, Dhib-Jalbut S, Hobart J, Kobelt G, Pepper G, et al. Brain health: time matters in multiple sclerosis. Mult Scler Relat Disord. 2016;9(Suppl 1):S5-48. https://doi. org/10.1016/j.msard.2016.07.003.

7. GMS Collaborators. Global, regional, and national burden of multiple sclerosis 1990-2016: a systematic analysis for the Global 
Burden of Disease Study 2016. Lancet Neurol. 2019. https://doi. org/10.1016/s1474-4422(18)30443-5.

8. Confavreux C, Vukusic S. The clinical course of multiple sclerosis. Handb Clin Neurol. 2014;122:343-69. https://doi. org/10.1016/B978-0-444-52001-2.00014-5.

9. Ahlgren C, Oden A, Lycke J. High nationwide prevalence of multiple sclerosis in Sweden. Mult Scler. 2011;17(8):901-8. https:// doi.org/10.1177/1352458511403794.

10. Thompson AJ, Banwell BL, Barkhof F, Carroll WM, Coetzee T, Comi G, et al. Diagnosis of multiple sclerosis: 2017 revisions of the McDonald criteria. Lancet Neurol. 2018;17(2):162-73. https ://doi.org/10.1016/S1474-4422(17)30470-2.

11. McNicholas N, Hutchinson M, McGuigan C, Chataway J. 2017 McDonald diagnostic criteria: a review of the evidence. Mult Scler Relat Disord. 2018;24:48-54. https://doi.org/10.1016/j. msard.2018.05.011.

12. Milo R, Miller A. Revised diagnostic criteria of multiple sclerosis. Autoimmun Rev. 2014;13(4-5):518-24. https://doi.org/10.1016/j. autrev.2014.01.012.

13. Solomon AJ, Naismith RT, Cross AH. Misdiagnosis of multiple sclerosis: impact of the 2017 McDonald criteria on clinical practice. Neurology. 2019;92(1):26-33. https://doi.org/10.1212/ wnl.0000000000006583.

14. Brownlee WJ, Swanton JK, Altmann DR, Ciccarelli O, Miller DH. Earlier and more frequent diagnosis of multiple sclerosis using the McDonald criteria. J Neurol Neurosurg Psychiatry. 2015;86(5):584-5. https://doi.org/10.1136/jnnp-2014-308675.

15. Brownlee WJ. Misdiagnosis of multiple sclerosis: if you have a hammer, everything looks like a nail? Neurology. 2019;92(1)1516. https://doi.org/10.1212/wnl.0000000000006584.

16. Culpepper WJ, Marrie RA, Langer-Gould A, Wallin MT, Campbell JD, Nelson LM, et al. Validation of an algorithm for identifying MS cases in administrative health claims datasets. Neurology. 2019;92(10):e1016-1028. https://doi.org/10.1212/wnl.00000 00000007043.

17. Wallin MT, Culpepper WJ, Campbell JD, Nelson LM, LangerGould A, Marrie RA, et al. The prevalence of MS in the United States: a population-based estimate using health claims data. Neurology. 2019;92(10):e1029-1040. https://doi.org/10.1212/ wnl.0000000000007035.

18. Widdifield J, Ivers NM, Young J, Green D, Jaakkimainen L, Butt DA, et al. Development and validation of an administrative data algorithm to estimate the disease burden and epidemiology of multiple sclerosis in Ontario, Canada. Mult Scler. 2015;21(8):1045-54. https://doi.org/10.1177/1352458514556303.

19. Ludvigsson JF, Otterblad-Olausson P, Pettersson BU, Ekbom A. The Swedish personal identity number: possibilities and pitfalls in healthcare and medical research. Eur J Epidemiol. 2009;24(11):659-67. https://doi.org/10.1007/s10654-009-9350-y.

20. The National Patient Register. National Board of Health and Welfare (Socialstyrelsen). 2018. http://www.socialstyrelsen.se/regis ter/halsodataregister/patientregistret/inenglish. Accessed 30 April 2018.

21. Wettermark B, Hammar N, Fored CM, Leimanis A, Otterblad Olausson P, Bergman U, et al. The new Swedish Prescribed Drug Register-opportunities for pharmacoepidemiological research and experience from the first six months. Pharmacoepidemioland Drug Saf. 2007;16(7):726-735. https://doi.org/10.1002/pds.1294.
22. The Cause of Death Register (Dödsorsaksregistret (in Swedish)). National Board of Health and Welfare (Socialstyrelsen). 2018. http://www.socialstyrelsen.se/register/dodsorsaksregistret\#. Accessed 30 April 2018.

23. Swedish Neuro Registry. Swedish Neuro Registries: Multiple Sclerosis. 2018. http://www.neuroreg.se/en.html/multiple-scler osis. Accessed 30 April 2018.

24. Hillert J, Stawiarz L. The Swedish MS registry-clinical support tool and scientific resource. Acta Neurol Scand. 2015;132(199):11-9. https://doi.org/10.1111/ane.12425.

25. Alping P, Piehl F, Langer-Gould A, Frisell T. Validation of the Swedish Multiple Sclerosis Register: further improving a resource for pharmacoepidemiologic evaluations. Epidemiology. 2019;30(2):230-3. https://doi.org/10.1097/ede.000000000000094 8.

26. Appendices to Open Comparisons of Healthcare Quality and Efficiency 2009: Indicator Descriptions, Coverage Comparison, and Health Consumption (Bilagor till Öppna jämförelser av hälso- och sjukvårdens kvalitet och effektivitet 2009: Indikatorbeskrivningar, Täckningsgradsjämförelser, and Vårdkonsumtion (in Swedish)). Stockholm: Swedish Association of Local Authorities and Regions and National Board of Health and Welfare (Sveriges Kommuner och Landsting och Socialstyrelsen); 2009.

27. MiDAS: Sjukpenning och rehabiliteringspenning. (MiDAS: sickness benefit and rehabilitation benefit.) (in Swedish). Social Insurance Agency (Försäkringskassan). 2011. www.forsakringskass an.se.

28. Longitudinal integration database for sick leave and labour market studies (LISA) 1990-2013. (Longitudinell integrationsdatabas för sjukförsäkrings- och arbetsmarknadsstudier (LISA) 1990-2013 (in Swedish)). Örebro: Statistics Sweden (Statistika centralbyrån); 2016.

29. Ludvigsson JF, Svedberg P, Olen O, Bruze G, Neovius M. The longitudinal integrated database for health insurance and labour market studies (LISA) and its use in medical research. Eur J Epidemiol. 2019;34(4):423-37. https://doi.org/10.1007/s10654-01900511-8.

30. Benchimol EI, Manuel DG, To T, Griffiths AM, Rabeneck L, Guttmann A. Development and use of reporting guidelines for assessing the quality of validation studies of health administrative data. J Clin Epidemiol. 2011;64(8):821-9. https://doi. org/10.1016/j.jclinepi.2010.10.006.

31. Foulon S, Maura G, Dalichampt M, Alla F, Debouverie M, Moreau T, et al. Prevalence and mortality of patients with multiple sclerosis in France in 2012: a study based on French health insurance data. J Neurol. 2017;264(6):1185-92. https://doi. org/10.1007/s00415-017-8513-0.

32. National guidelines Care for multiple sclerosis and Parkinson's disease: support for steering and management (Nationella riktlinjer Vård vid multipel skleros och Parkinsons sjukdom: Stöd för styrning och ledning (in Swedish)). Falun: National Board of Health and Welfare (Socialstyrelsen); 2016.

Publisher's Note Springer Nature remains neutral with regard to jurisdictional claims in published maps and institutional affiliations. 